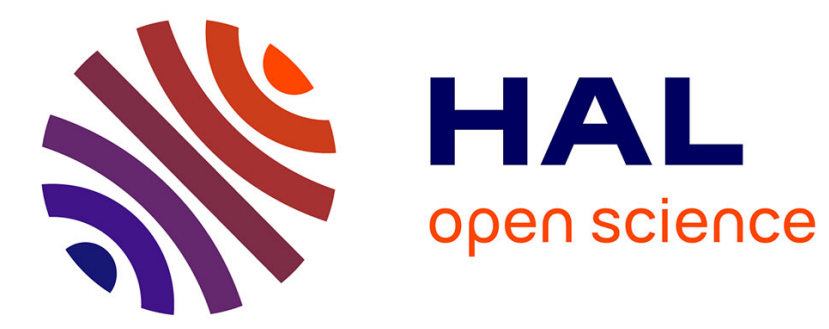

\title{
Physical processes in proximity microbridges
}

\author{
J.E. Mercereau
}

\section{To cite this version:}

J.E. Mercereau. Physical processes in proximity microbridges. Revue de Physique Appliquée, 1974, 9

(1), pp.47-52. 10.1051/rphysap:019740090104700 . jpa-00243773

\section{HAL Id: jpa-00243773 https://hal.science/jpa-00243773}

Submitted on 1 Jan 1974

HAL is a multi-disciplinary open access archive for the deposit and dissemination of scientific research documents, whether they are published or not. The documents may come from teaching and research institutions in France or abroad, or from public or private research centers.
L'archive ouverte pluridisciplinaire HAL, est destinée au dépôt et à la diffusion de documents scientifiques de niveau recherche, publiés ou non, émanant des établissements d'enseignement et de recherche français ou étrangers, des laboratoires publics ou privés. 


\title{
PHYSICAL PROCESSES IN PROXIMITY MICROBRIDGES
}

\author{
J. E. MERCEREAU
}

\author{
California Institute of Technology, 63-37, Pasadena, California 91109, USA
}

\begin{abstract}
Résumé. - Des circuits supraconducteurs utilisant les effets de proximité et qui présentent des effets "Josephson » dans une large gamme de variation des paramètres électriques et physiques ont été développés. Les caractéristiques de ces circuits sont discutées et un modèle physique simple qui rend compte de la modification des phénomènes aux fortes densités de courant est analysé. La réponse de ses dispositifs aux hautes fréquences est étudiée en mettant l'accent sur les processus de détection et l'effet Dayem-Wyatt.
\end{abstract}

\begin{abstract}
Weakly superconducting circuits have been developed - based on the proximity effect in hard superconductors - which show Josephson-like phenomena over a wide range of electrical and superconducting parameters. Characteristics of these circuits will be discussed and a simple physical model will be presented to account for a modification of the Josephson phenomena at high current density in these devices. Response of the devices at high frequency will be emphasized with special emphasis on detection processes and the Dayem-Wyatt effect.
\end{abstract}

1. Introduction. - The concept of macroscopic quantum interference was first introduced by Fritz London before 1935 [1]. But it was not until 1962 when Brian Josephson [2] refined the idea and applied it to describe the supercurrent produced by the tunneling of electron pairs between two weakly connected superconductors that the full impact of the phenomenon became apparent. In subsequent years, this idea has had considerable experimental investigation and Josephson effects have been observed in a wide variety of weakly connected superconducting circuits. This paper is concerned with a particular type of weakly connected superconducting thin film circuit (or microbridge) within which the strength of coupling can be manipulated as an experimental parameter. The circuits are formed of contiguous layered superconducting films within which the transition temperature can be locally modified by means of the proximity effect.

Since these are conducting circuits with a well defined normal resistance, $R$, we have found it expedient to describe the electrical response of the circuit primarily in terms of oscillating "Josephson potential» [3] rather than oscillating Josephson current. In fact, within these conducting microbridges, the total current is conserved but, on a two fluid model, the supercurrent component undergoes a relaxation oscillation at the Josephson frequency. Since the total current is conserved, the oscillating supercurrent component is reflected in a time dependent potential $v_{\mathrm{s}}(t)$, developed across the microbridge where, $v_{\mathrm{s}}(t)=R I_{\mathrm{s}}(t)$. In this non-equilibrium situation, the effective chemical potential for the quasi-particles may differ significantly from the effective chemical potential for the pairs [4].

These proximity effect microbridges are essentially small regions within a superconducting film, across which it is possible to develop differences in chemical potential, and within which the amplitude and phase of the macroscopic quantum wave function are themselves functions of both space and time.

2. Fabrication. - In the past few years, we have developed general techniques for the fabrication of superconducting thin film structures containing well defined inhomogeneities in the amplitude of the superconducting wave function. The Josephson-like characteristics of these structures have been investigated over a wide range of electrical and materials parameters [5]. The techniques are based on the use of the proximity effect to locally modify the relative transition temperature, and hence the amplitude or strength of the superconducting wave function, between various parts of a composite superconducting film.

When a normal film is superimposed directly on a superconducting film with no intervening oxide layer or vice versa, the transition temperature of the resultant superconducting sandwich is depressed below that of the superconductor itself. In the thin film limit, the transition temperature of the sandwich depends on the relative thicknesses of the normal and superconducting films and decreases as the normal material is made thicker. A similar situation exists for a sandwich of two superconductors of different transition temperature. This composite thin film 
material we call a superconducting thin film substrate. Since the transition temperature is a measure of the relative strength of the superconductivity, as the transition temperature decreases the weaker the superconducting sandwich film becomes. Hence by locally varying the relative thicknesses of superimposed normal and superconducting films, it is possible to develop spatial variations in the amplitude of superconducting wave function within the thin film substrate.

In this way a thin film structure of the form in figure 1 can be constructed where the short inhomogeneous region of length $l$, width $w$, and thickness $t$, has a characteristic transition temperature $T_{\mathrm{c}}^{\prime}$ lower than the transition temperature $T_{\mathrm{c}}$ of the adjoining film. It is thus possible to vary the coupling strength between the main superconducting film sections by varying the $T_{\mathrm{c}}^{\prime}$ and the length $l$. We have fabricated these thin film structures from many different superconducting materials and have always found Josephson-like characteristics for an appropriate combination of $T_{\mathrm{c}}^{\prime}$ and $l$. Results reported here are typical of all such structures which we have examined, but in particular are for a substrate formed of niobium on tantalum. Photoresist techniques are used to define the superconducting films and anodizing or ion etch techniques have been used to control the film thickness [6].

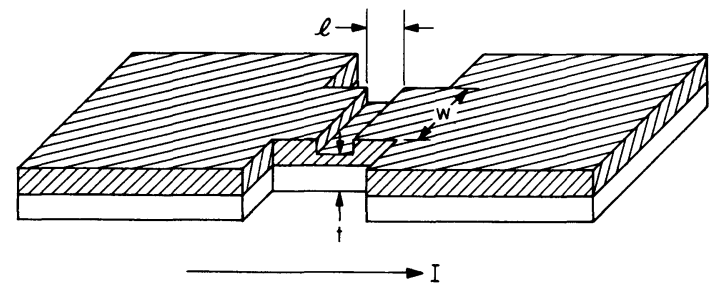

FIG. 1. - Schematic drawing of the two layer thin film substrate ( $\mathrm{Nb}$ on $\mathrm{Ta}$ ) discussed in the text. The thin region has a lower transition temperature $T_{\mathrm{c}}^{\prime}$ than the adjoining superconductors. Current flows in the direction noted $I$.

It should be emphasized that it is the spatial modulation of the wave function which is important in this application. These substrates have been developed so that experimentally controllable changes in the thickness of the films results in the controllable spatial modulation of the wave function. In contrast with other forms of weak link [7], it is not the smallness of the bridge itself which is critical in this application. In fact, in many of our configurations, the weak section is actually thicker than the surrounding material.

3. Experimental results. - Typical electrical characteristics for these microbridges are shown in figure 2 . The average potential $\bar{V}$ and $\mathrm{rf}$ potential $v_{\mathrm{s}}(t)$ are measured across a weakened section, such as we have described above, in response to passing a current $I$ in excess of the critical current $I_{c}$ through

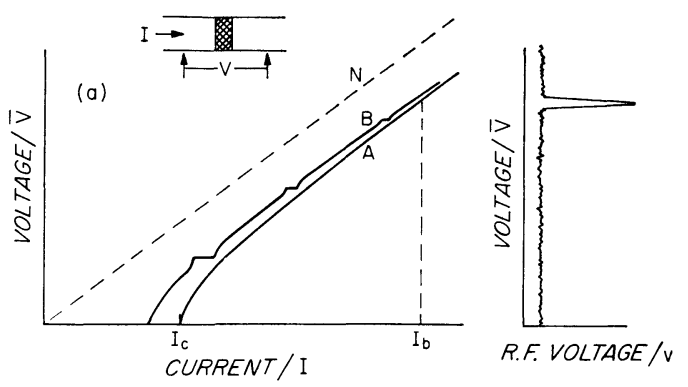

FIG. 2a. - Left : Current-voltage $I-\bar{V}$, characteristics for zero magnetic field. Curve $\mathrm{N}$ is for $T>T_{\mathrm{c}}^{\prime}$, bridge in the normal state ; curve $\mathrm{A}$ is for $T<T_{\mathrm{c}}^{\prime}$; curve $\mathrm{B}$ shows the effect of radiation. Right : Oscillating potential $v_{\mathrm{S}}(t)$ as a function of frequency $\Omega=2 \mathrm{e} \bar{V} / \hbar$ for a bridge current biased at $I_{\mathrm{b}}$.

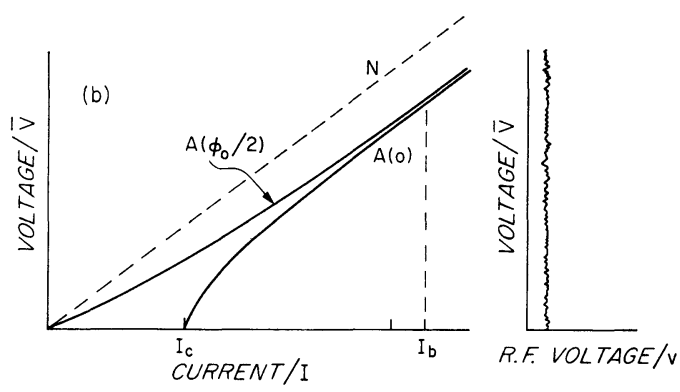

FIG. $2 b$. - Effects of magnetic field on $I-\bar{V}$ characteristics. Curve $A(0)$ is for zero field, curve $A\left(\varphi_{0} / 2\right)$ is for flux $\varphi_{0} / 2$ in bridge region. At this flux level no radiation induced steps or oscillating potential $v_{\mathrm{S}}(t)$ are observed.

the junction. As long as the critical current is insufficient to support a flux quantum $\left(\varphi_{0}=h / 2 \mathrm{e}\right)$ within the bridge $\left(I_{\mathrm{c}} \lesssim \varphi_{0} / \mu_{0} w\right)$ the $I-\bar{V}$ curves show no hysteresis. Figure $2 a$ represents a typical $I-\bar{V}$ curve in this limit in zero magnetic field. The trace labeled $N$, for which $V_{\mathrm{n}}=I R$, is for a situation in which the bridge section is normal while the adjoining films are superconducting. The resistance $R$, measured in this way is not a particularly strong function of temperature, but does decrease slightly as the temperature is decreased due to proximity effects across the bridge. Nevertheless, the resistance of these bridges is an easily measurable experimental parameter. The trace labeled $\mathrm{A}$ is for a temperature at which the bridge section carries supercurrent. In this situation, potential developed across the bridge is always less than the potential of the normal state $V_{\mathrm{n}}$ and at high voltages $\left(I \gg I_{\mathrm{c}}\right)$ tends to be less than $V_{\mathrm{n}}$ by a constant, $R I_{\mathrm{c}} / 2$.

The right hand side of figure $2 a$ represents the radio frequency potential measured across a bridge biased at a fixed current $I_{\mathrm{b}}$. This potential oscillates at the Josephson frequency and if $I_{b}$ is large compared to the critical current, the oscillation is nearly harmonic and the amplitude of the oscillating potential is $R I_{\mathrm{c}} / 2$. The trace labeled $\mathrm{B}$ represents the time average potential developed across the bridge simultaneously biased with both constant current and high frequency current induced by an incident microwave 
field. The effects of the high frequency currents are to slightly suppress the $I-\bar{V}$ curve from the unirradiated situation and to form "steps » at voltages corresponding to the fundamental, harmonics and subharmonics of the exciting frequency.

Figure $2 b$ represents the effects of a magnetic field on the potential developed across a current biased bridge. The curve labeled $\mathrm{A}(0)$ represents a superconducting $I-\bar{V}$ curve in zero magnetic field similar to curve $\mathrm{A}$ in figure $2 a$. The curve labeled $\mathrm{A}\left(\varphi_{0} / 2\right)$ represents the $I-\bar{V}$ curve in a magnetic field corresponding to one half of a flux quantum in the weak region. The critical current has been suppressed to zero, and at finite current the potential is increased from the zero field situation but approaches $V_{\mathrm{n}}-R I_{\mathrm{c}} / 2$ for $I$ is much larger than $I_{\mathrm{c}}$. For this magnetic field, the oscillating potential $v_{\mathrm{s}}(t)$ disappears and the response of the bridge to external radiation ceases. For this particular magnetic field the step structure of the $I-\bar{V}$ curve, such as that induced in curve B, figure $2 a$ vanishes completely.

As a function of magnetic field, the response of a bridge varies smoothly between the extremes illustrated by figures $2 a$ and $2 b$. The period of this modulation corresponds to a flux quantum in the area of the bridge and the amplitude of the modulation tends to decrease with increasing magnetic field in a manner reminiscent of the magnetic field modulation of the dc critical current in a Josephson tunnel junction.

4. Physical model. - A physical model for this type of superconductivity has been developed based on a relaxation oscillation [8] in the amplitude of the superconducting wave function, at the Josephson frequency. During the relaxation oscillation, the total current is conserved which leads to an oscillation in potential across the weak region where $v_{\mathrm{s}}(t)=R I_{\mathrm{s}}(t)$. Many of the characteristics of this relaxation oscillation or "phase slip " have been calculated [9] and the results are consistent with a phenomenological two fluid model in which, above the critical current of the bridge, the supercurrent equals,

$$
I_{\mathrm{s}}=\left(\frac{I_{\mathrm{c}}}{2}\right)\left(1+\cos \frac{2 \mathrm{e}}{\hbar} \int V \mathrm{~d} t\right) .
$$

Thus in the one dimensional limit (uniform distribution of current and no flux vortices) the potential across the bridge, as indicated by the insert in figure $2 a$, is given by

$$
V=I R-v_{\mathrm{s}}\left(1+\cos \frac{2 \mathrm{e}}{\hbar} \int V \mathrm{~d} t\right),
$$

where we write $v_{\mathrm{s}}=R I_{\mathrm{c}} / 2$. The asymmetry of the supercurrent relative to zero reflects the fact that there is an intrinsic dissipation associated with the relaxation oscillation. This particular analytic form for the potential is consistent with the shape of the
$I-\bar{V}$ curves as shown in figure 2 as well as the measured amplitude of the oscillating potential $v_{\mathrm{s}}(t)$.

In this mode, the application of a magnetic field induces circulating currents within the junction, and a field equivalent to $\frac{1}{2}$ quantum in the junction produces a circulating current sufficiently large to suppress the critical current to zero. At a finite voltage this "vortex » state also oscillates at the Josephson frequency. The supercurrent at opposite ends of the junction oscillates at the Josephson frequency, as in eq. (1) but $\pi$ out of phase. In this kind of oscillation, the net supercurrent through the bridge is constant in time and produces no oscillating longitudinal potential $v_{\mathrm{s}}(t)$ across the bridge. However, since the supercurrent at opposite ends of the bridge is out of phase, an oscillating transverse potential (perpendicular to the direction of current $I$ ) will be developed between the ends of the bridge.

Dissipation associated with this transverse potential oscillation shows up as an increased $\bar{V}$ potential for a given current such as in figure $2 b$. The transverse oscillation will dissipate more power than the corresponding longitudinal oscillation, due to the transverse oscillating potential, $V_{\mathrm{T}}$ (which exists from end to end of the resistive bridge in this mode) by an amount

$$
\frac{V_{\mathrm{T}}^{2}}{2 R_{\mathrm{T}}}=\frac{2 R^{2} I_{\mathrm{c}}^{2}}{R_{\mathrm{T}}}
$$

This extra power must be supplied from the current source and shows up as an increased voltage $\delta V$, over the longitudinal oscillation, where $I \delta V=2 R^{2} I_{\mathrm{c}}^{2} / R_{\mathrm{T}}$ for a given current bias $I$. This approximation describes the voltage displacement between $\mathrm{A}(0)$ and $\mathrm{A}(\varphi / 2)$, figure $2 b$, for current large relative to the critical current, but fails at lower currents due to the nonharmonic character of the oscillation in this regime. The absence of a longitudinal oscillation in potential for this particular magnetic field indicates that there should be no coupling of the junction to rf longitudinal currents such as would be produced in the junction by a radiation field.

These bridges, therefore, can be characterized as current controlled voltage oscillators, oscillating at the Josephson frequency where the phase of the oscillation is a function of magnetic field. The effects of radiation induced $\mathrm{rf}$ currents on these voltage oscillators can be determined by an examination of eq. (2). Solutions for equations of this type have been discussed elsewhere [10] and here we will emphasize only the physical processes underlying the mathematics. We treat here the longitudinal oscillation for which the magnetic field is zero. Without radiation induced currents, eq. (2) can be solved exactly for the time average potential $\bar{V}_{\mathrm{e}}$ developed by a current $I$ in excess of the critical current $I_{c}$. This solution is

$$
\bar{V}_{\mathrm{e}}=R I\left(1-\frac{2 v_{\mathrm{s}}}{R I}\right)^{1 / 2},
$$


and is consistent with all of our experimental results on this type of bridge in the low current limit. The time dependent potential has also been calculated [11] from eq. (2) and produces a wave form which also is consistent with experimental results.

When the bias current $I_{\mathrm{b}}$ is large compared to the critical current, the potential developed across the bridge can be approximated as

$$
V \simeq \bar{V}-v_{\mathrm{s}} \cos \frac{2 \mathrm{e}}{\hbar} \bar{V} t
$$

In this approximation, the time average voltage $\bar{V}$ is $\left(I_{\mathrm{b}} R-v_{\mathrm{s}}\right)$ and the time dependent voltage has an amplitude $v_{\mathrm{s}}=R I_{\mathrm{c}} / 2$ and frequency $\Omega=2 \mathrm{e} \bar{V} / \hbar$. Therefore, in first approximation, the effect of induced rf currents, $I_{\mathrm{rf}}$, through the bridge at frequency $\omega$ is to modify this potential to become

$$
V_{1}=\bar{V}-v_{\mathrm{s}} \cos \Omega t+I_{\mathrm{rf}} R \sin \omega t .
$$

To obtain a second approximation, the voltage, $V_{1}$, can be used to determine the frequency in eq. (2)

$$
V_{2}=I R-v_{\mathrm{s}}\left(1+\cos \frac{2 \mathrm{e}}{\hbar} \int V_{1} \mathrm{~d} t\right) .
$$

The time average of this equation for $V_{2}$ is :

$$
\bar{V}_{2}=\bar{V}-v_{\mathrm{s}} J_{0}\left(\frac{2 \mathrm{e} v_{\mathrm{rf}}}{\hbar \omega}\right) J_{1}\left(\frac{v_{\mathrm{s}}}{\bar{V}}\right),
$$

where we have written $v_{\mathrm{rf}}=R I_{\mathrm{rf}}$ and $J$ represents a Bessel function. When the radiation field is zero, $v_{\text {rf }}=0$, eq. (5) reduces to $\bar{V}_{2}=\bar{V}-v_{\mathrm{s}} J_{1}\left(v_{\mathrm{s}} / \bar{V}\right)$ which is a good approximation to the exact solution, eq. (3) in this high current limit. Therefore, the general effect of the radiation field in modifying the $I-\bar{V}$ curve is to increase the potential at a given current by an amount

$$
\delta \bar{V}_{2}=v_{\mathrm{s}} J_{1}\left(\frac{v_{\mathrm{s}}}{\bar{V}}\right)\left[1-J_{0}\left(\frac{2 \mathrm{e} v_{\mathrm{rf}}}{\hbar \omega}\right)\right] .
$$

At low $\mathrm{rf}$ power levels and for $\bar{V}>v_{\mathrm{s}}$ this simplifies to :

$$
\delta V \simeq\left(\frac{v_{\mathrm{s}}}{8}\right)\left(\frac{v_{\mathrm{s}}}{\bar{V}}\right)\left(\frac{2 \mathrm{e} v_{\mathrm{rf}}}{\hbar \omega}\right)^{2} .
$$

Thus at low rf power in this model, the $I-\bar{V}$ curve is altered by a general increase in potential which is proportional to $\mathrm{rf}$ power and decreases with increasing $\bar{V}$, similar to the effect indicated in the experimental data shown as curve $\mathbf{B}$, figure $2 a$.

The average potential developed across the bridge also indicates the average frequency of the quantum oscillator. As we have shown, the general effect of an applied radiation field is to slightly increase the voltage (or oscillation frequency) at a given current. However, when the radiation frequency is equal to the natural frequency of the oscillator, the oscillator tends to synchronize to the radiation field and tends to remain locked at the radiation frequency for a considerable range of current. In other words, current « steps » are developed. The mechanism for this locking is also contained in eq. (4). When the oscillator and radiation frequencies are the same, the time average potential depends on the relative phase between the oscillator and the radiation field. The stable condition is when the oscillator lags behind the radiation field by $\pi / 2$; lagging further behind increases the time average potential which therefore increases the frequency of the oscillator to bring it back to synchronism. Conversely, as the phase angle becomes less than $\pi / 2$ the time average voltage decreases allowing the oscillator to slow down.

The size of the current step predicted by this model is related to the excursion in potential $\delta \vec{V}$ (and therefore the excursion in oscillator frequency) produced by a radiation field at frequency $\omega$ close to the natural frequency, $\Omega$, of the oscillator. Solving eq. (4) in the low power limit, $2 \mathrm{e} v_{\text {rf }} \ll \hbar \omega,|\Omega-\omega| \ll \Omega$, the oscillator frequency changes in time, or hunts, at a rate proportional to $(\Omega-\omega)$ over a frequency range

$$
\Delta \omega \sim \frac{2 \mathrm{e} v_{\mathrm{s}}}{\hbar} J_{0}\left(\frac{v_{\mathrm{s}}}{\bar{V}}\right) J_{1}\left(\frac{2 \mathrm{e} v_{\mathrm{rf}}}{\hbar \omega}\right) \text {. }
$$

When $|\Omega-\omega|$ becomes less than $\Delta \omega$, the hunting ceases and the oscillator synchronizes to the radiation frequency. The voltage range over which the oscillator can sweep for the $n$th step is

$$
\left(\delta V_{\mathrm{n}}\right) \sim v_{\mathrm{s}} \times J_{0}\left(\frac{v_{\mathrm{s}}}{\bar{V}}\right) J_{n}\left(\frac{2 \mathrm{e} v_{\mathrm{rf}}}{\hbar \omega}\right) .
$$

Thus a current step in this approximation is

$$
\delta I_{n} \sim R^{-1} \delta V_{n} \sim I_{\mathrm{c}} J_{0}\left(\frac{v_{\mathrm{s}}}{\bar{V}}\right) J_{n}\left(\frac{2 \mathrm{e} v_{\mathrm{rf}}}{\hbar \omega}\right) .
$$

In general, experimental results on this type of junction tends to conform quite well to predictions based on this model.

5. Analysis. - The physical basis for this model is the process of phase slip, which is a time dependent, non-equilibrium state of superconductivity. In this state, the amplitude of the superconducting wave function periodically becomes unstable and decays to zero. The assumption of phase slip is that the system subsequently recovers into a phase coherent state in which the relative phase difference between any two points separated by the slip region has changed by $2 \pi$. A detailed calculation based on a time dependent Ginzburg-Landau theory has been done [9] and leads to a current-voltage relationship very similar to eq. (2).

An attempt has also been made to describe this non-equilibrium situation in terms of a two component wave function [13], whose amplitudes are not time dependent, but whose relative phase is governed by the Josephson relation. This model reproduces most 
of the results of phase slip and predicts some new effects related to the phase gradient. Figure $3 a$ shows schematically the equilibrium amplitude of the wave function as a function of position through the weakened bridge region. By "weakening» the superconductivity in the bridge area, the amplitude is depressed below that in the adjoining superconductor. When a current flows through the bridge $\left(j \propto \psi^{2} \nabla \varphi\right)$, the phase becomes position dependent such as indicated to the right of figure $3 a$. The phase gradient increases in the weak region to compensate for the decrease in $\psi^{2}$ in that region. Out of equilibrium (see Fig. $3 b$ ), we assume that the wave function can be expressed in terms of two separate, independent parts $\Psi=\psi_{1}+\psi_{2}: \psi_{1}$ in quasi-equilibrium with the superconductor on the left and $\psi_{2}$ in quasi-equilibrium with the superconductor on the right. The amplitudes of the separate wave functions in the weak area

$$
\left(\psi_{1}=f(x) \mathrm{e}^{i \varphi_{1}(x, t)} \quad \text { and } \quad \psi_{2}=g(x) \mathrm{e}^{i \varphi_{2}(x, t)}\right)
$$
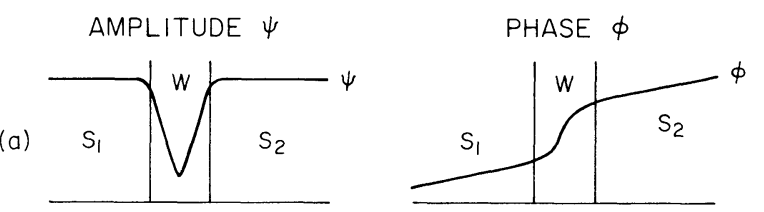

FIG. $3 a$. - Schematic representation of the wave function, amplitude (left) and phase (right) for a current carrying bridge in equilibrium.

(b)
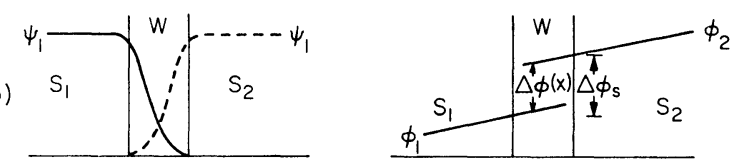

FIG. $3 b$. - Schematic representation of a non-equilibrium situation in which the wave function is described in terms of two overlapping parts, amplitude (left) and phase (right).

POTENTIAL

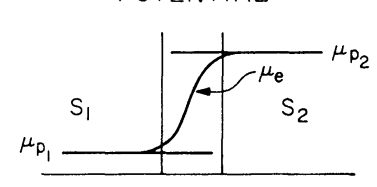

FIG. $3 c$. - Schematic representation of the instantaneous chemical potentials near a voltage supporting bridge. The chemical potential for pairs $\left(\mu_{\mathrm{p}_{1}}\right.$ and $\left.\mu_{\mathrm{p}_{2}}\right)$ and electrons $\mu_{\mathrm{e}}$ are all different.

are assumed to be time independent and determined by proximity effects relative to the appropriate adjoining superconductor. The simplest current carrying non-equilibrium state of this type can be represented by the phase relationship illustrated to the right of figure $3 b$. If a potential $V$ is developed across the weak section, such as illustrated in figure $3 c$, then $2 \mathrm{e} V=\mu_{\mathrm{p}_{1}}-\mu_{\mathrm{p}_{2}}$, and the relative phase between these two wave functions will be determined by the Joseph- son relationship. Because of interference effects between the two wave functions $\psi_{1}$ and $\psi_{2}$, the amplitude of the composite wave function $\Psi$ will then be a function of time.

Given this wave function, current can be calculated from the usual operator

$$
j_{\mathrm{s}}=\operatorname{Re}\left(\Psi\left(\frac{\mathrm{e} \hbar}{i m}\right) \nabla \Psi\right),
$$

the result is

$$
\begin{aligned}
j_{\mathrm{s}}=\frac{\mathrm{e} \hbar}{m}\left[(f \nabla g-g \nabla f) \sin \left(\varphi_{2}-\varphi_{1}\right)+f^{2} \nabla \varphi_{1}+\right. \\
\left.+g^{2} \nabla \varphi_{2}+f g\left(\nabla \varphi_{1}+\nabla \varphi_{2}\right) \cos \left(\varphi_{2}-\varphi_{1}\right)\right]
\end{aligned}
$$

When the phase gradient can be neglected, this approach is just the Aslamazov-Larkin [14] derivation of the Josephson effect. However, in situations of high current density (or large phase gradients, $\nabla \varphi$ ) the second term may become important and even predominate.

The second term is similar to our eq. (1), with some important differences. In the $\sin \left(\varphi_{2}-\varphi_{1}\right)$ term of eq. (6), the gradients of $f$ and $g$ are constants, fixed by the spatial variation of $f$ and $g$. Thus the amplitude of the sine term is a constant, determined by the spatial decay of the separate wave functions. However, in the second term, the gradient of the phase is not a constant and must be determined by the external boundary conditions on current and field. Thus the amplitude of the second term is not necessarily constant and the phase dependence of this term must be considered carefully.

We will indicate two limiting features of this current : the existence of a critical current and the response to a voltage source. The second part of eq. (6) can be simplified further if we assume that momentum is conserved in going from $\psi_{1}$ to $\psi_{2}$, or that $\nabla \varphi_{1}=\nabla \varphi_{2}$ as illustrated to the right of figure $3 b$ where phase is plotted as a function of position through the weak section. In order to illustrate the physics, we evaluate eq. (6) at the point of maximum overlap where $f(x)=g(x)=f_{0}$ and the current becomes:

$$
j=2 f_{0}^{2} \nabla \varphi\left[1+\cos \left(\varphi_{2}-\varphi_{1}\right)\right] .
$$

The phase difference $\varphi_{2}-\varphi_{1}=\Delta \varphi(x)$ is also a constant in this situation and is related to the phase difference across the weak section,

$$
\Delta \varphi(s) \text { by } \Delta \varphi(x)=\Delta \varphi(s)-(\nabla \varphi) l
$$

as illustrated in figure $3 b$. Maximum current occurs when $\cos =1$, or when $\nabla \varphi=\Delta \varphi_{\mathrm{s}} / l$. Since the maximum phase difference between $S_{1}$ and $S_{2}$ is $2 \pi$, $(\nabla \varphi)_{\max }=2 \pi / l$. Thus eq. (7) will have a critical current of amplitude $j_{\mathrm{c}}=f_{0}^{2} / \pi l$. If $f$ decays exponentially at a rate $\xi_{n} \sim \hbar v_{\mathrm{f}} / k T$ into the weak region, the amplitude of this critical current then has the same func- 
tional form as that calculated by Bardeen and Johnson [12] for a $\mathrm{S}|\mathrm{N}| \mathrm{S}$ Josephson junction. This exponential dependence of critical current with temperature is consistent with experimental results [5] for these bridges. Applied current above this critical value will develop a normal component and a potential distribution such as illustrated in figure $3 c$. The phase difference will then be governed by the Josephson relation

$$
\left(\varphi_{2}-\varphi_{1}\right)=\left(\frac{1}{\hbar}\right) \int\left(\mu_{\mathrm{p}_{1}}-\mu_{\mathrm{p}_{2}}\right) \mathrm{d} t
$$

and for $j>j_{\mathrm{c}}$ this model predicts a time dependent supercurrent,

$$
j=\frac{f_{0}^{2}}{\pi l}\left[1+\cos \frac{2 \mathrm{e}}{\hbar} \int V \mathrm{~d} t\right],
$$

similar to eq. (1) and the phase slip model.

Below the critical current the phase gradient is determined by boundary conditions imposed by the currents and fields. The phase gradient is proportional to the supercurrent and vector potential such that $\nabla \varphi=\int E \mathrm{~d} t+\alpha$ where $E$ is the electric field and $\alpha$ is a constant to determine the supercurrent when the field is zero. Thus

$$
\begin{aligned}
\Delta \varphi(x) & =\Delta \varphi(s)-\frac{2 \mathrm{e}}{\hbar} \int(E l) \mathrm{d} t \\
& =\Delta \varphi(s)-\frac{2 \mathrm{e}}{\hbar} \int V \mathrm{~d} t
\end{aligned}
$$

and in this limit eq. (7) becomes,

$j \propto\left(\int E \mathrm{~d} t+\alpha\right)\left[1+\cos \left(\frac{2 \mathrm{e}}{\hbar} \int V \mathrm{~d} t+\beta\right)\right]$,

where $\beta$ is the phase difference across the bridge at zero voltage. This supercurrent is time dependent both through the amplitude and the phase difference. These two terms can beat against each other and one of the predictions of this analysis is a rectified supercurrent produced from high frequency radiation. This effect has been suggested [13] as a possible explanation of the Dayem-Wyatt phenomenon which is particularly pronounced in these proximity effect bridges.

This analysis is essentially based on a speculation that these are non equilibrium conditions in which the discontinuous phase slip description can be replaced by a quasi equilibrium process as represented by $\Psi=\psi_{1}+\psi_{2}$. It leads to the usual expression for Josephson current and transport current $\left(j_{\mathrm{t}} \propto \psi^{2} \nabla \varphi\right)$ except that the amplitude $\psi^{2}$ of the transport current is itself phase dependent. Many of the unusual characteristics of the proximity effect microbridges can be described in this way and the approach may be applicable to other types of non equilibrium superconducting junctions.

Acknowledgments. - The author wishes to thank Dr. H. A. Notarys for his valuable assistance and contributions. This work was supported by the Office of Naval Research, Contract no N00014-67-A-0094-0013 and the Jet Propulsion Laboratory, Director's Discretionary Fund.

\section{References}

[1] London, F., Proc. R. Soc. A 152 (1935) 24.

[2] Josephson, B. D., Phys. Rev. Lett. 1 (1962) 251.

[3] Notarys, H. A., Wang, R. H. and Mercereau, J. E., Proc. IEEE 61 (1973) 79.

[4] Rieger, T. J., Scalapino, D. J. and Mercereau, J. E., Phys. Rev. Lett. 27 (1971) 1787.

[5] Notarys, H. A. and Mercereau, J. E., J. Appl. Phys. 44 (1973) 1821.

[6] Palmer, D. W. and Decker, S. K., Rev. Sci. Instrum. November 1973.

[7] Anderson, P. W. and Dayem, A. H., Phys. Rev. Lett. 13 (1964) 195
[8] Notarys, H. A. and Mercereau, J. E., Proc. 1969 International Conference on Superconductivity (ed. F. Chilton North Holland, Amsterdam) 1971, p. 424.

[9] Rieger, T. J., Scalapino, D. J. and Mercereau, J. E., Phys. Rev. B 6 (1972) 1734.

[10] Kanter, H. and Vernon, F. L., J. Appl. Phys. 43 (1972) 3174.

[11] Kirschman, R. K., J. Low Temp. Phys. 11 (1973) 235.

[12] Bardeen, J. and Johnson, J. L., Phys. Rev. 5 (1972) 72.

[13] Notarys, H. A., Yu, M. L. and Mercereau, J. E., Phys. Rev. Lett. 30 (1973) 743.

[14] Wyatt, A. F. G., et al., Phys. Rev. Lett. 15 (1966) 1166. 